\title{
ORAL MICROBIOME OF PERMANENTLY MENTALLY DISABLED AND HEALTHY CHILDREN
}

\author{
Smatanova $\mathbf{M}^{1}$, Novakova $E^{2}$, Bacinsky $\mathbf{M}^{1}$, Hvizdos $D^{1}$, Statelova $\mathrm{D}^{1}$, Kompanikova $\mathrm{J}^{2}$, \\ NovaK $\mathrm{M}^{3}$, MIKUSKOVA $\mathrm{K}$. ${ }^{1}$ \\ ${ }^{1}$ Clinic of Stomatology and Maxillofacial Surgery, Jessenius Faculty of Medicine in Martin, \\ Comenius University in Bratislava, Martin, Slovakia \\ ${ }^{2}$ Institute of Microbiology and Immunology, Jessenius Faculty of Medicine in Martin, Comenius \\ University in Bratislava, Martin, Slovakia \\ ${ }^{3}$ Department of Public Health, Jessenius Faculty of Medicine in Martin, Comenius University in \\ Bratislava, Martin, Slovakia
}

\begin{abstract}
A b s t r a c t
The oral cavity is a biologically significant and complex site of the human body. It is a gateway into the internal environment of the organism. There are many processes, such as the beginning of digestion, speech creation, and sensory perception of taste. Oral health is closely related to the general health of a person. The oral cavity contains an enormous number of microorganisms that can cause various diseases. Oral bacteria are responsible for diseases in the mouth, but can also seriously harm human health. The oral microbiome also serves as an indicator of health, respectively morbidity of the human organism. Compared to healthy children, mentally disabled children suffer from many congenital and acquired diseases and disorders that affect their overall and oral health. These children require a specific approach to the examination, but also to the therapy.

Therefore, it is necessary to pay attention to the prevention of oral diseases in children, whether healthy or mentally disabled.
\end{abstract}

Key words: microbiome, healthy children, mentally disabled children

\section{INTRODUCTION}

By microbiome we mean all commensal, symbiotic, and pathogenic microorganisms that occur in the human body, such as bacteria, viruses, fungi, protozoa, archaea, and they together form an ecological community. These are the genomes of microorganisms in the human body. (1)

The human mouth is heavily colonized by microorganisms. Unlike the commensal microflora, which is found elsewhere in the body where it lives in harmony with the host, the normal oral flora is responsible for two most common human diseases - tooth decay and periodontal disease. The bacterial communities found in the mouth form a large complex with about 1,000 species. The amount of bacteria in the oral cavity is thus comparable to the colonization of the colon. This is due to the properties of the environment, especially the high content of readily available nutrients. $(2,3,4)$

The composition of the oral microbiome is influenced by various factors such as diet, oral hygiene, sugar consumption, the use of antibiotics and other antimicrobials. If there is an imbalance in the oral microbiome, caries, periodontal disease, or candidiasis occur and the oral bacteria begin to spread to otherwise sterile parts of the body and extraoral infections develop.

Bacterial population of the oral cavity changes over the course of a lifetime. (5)

Corresponding author: Katarína Mikušková, MD, PhD.; e-mail: katarina.mikuskova@uniba.sk

(C) 2020 Smatanova M. et al.

This work is licensed under the Creative Commons Attribution-NonCommercial-NoDerivs 4.0 License (https://creativecommons.org/licenses/by-nc-nd/4.0/) 


\section{ORAL MICROBIOME}

Some studies suggest that there are links between the placental environment and the oral microbiome. 16S rRNA gene sequencing analysis indicates the presence of certain oral bacteria in the human and mouse placenta, such as Streptococcus, Fusobacterium, Neisseria, Prevotella, and Porphyromonas. (6)

As the first colonizers of the newborn's oral cavity there are Lactobacilli, Bifidobacteria, and Streptoccocci which come from the birth canal, breast milk, and the mother's mouth. There is a difference in microbiome in breastfed infants and formula fed infants. A threemonth fully breastfed diet has oral lactobacilli with antimicrobial properties in contrast to a diet with artificial substitutes. Also the way a child is born affects the microbiome. For example, Streptococcus (S.) mutans was detected a year earlier in infants born by caesarean section than in infants born vaginally. (5) Children born vaginally have the oral microbiome similar to the mother's vaginal microbiome. In these children the following bacterial species can be found: Lactobacillus, Prevotella, Corynebacterium, Staphylococcus, Streptococcus, Enterococcus, Bacteroides, and Sneathia spp.

Children born via cesarean section have the oral microbiome similar to the mother's skin microbiome. In these children the following bacterial species can be found: Staphylococcus, Corynebacterium, Propionibacterium spp. belonging to the phylum such as Actinobacteria, Firmicutes, Proteobacteria, and Bacteroidetes. $(7,8)$

The newborn's oral microbiome is composed mainly of bacteria of these six phyla: Firmicutes, Proteobacteria, Actinobacteria, Bacteroidetes, Fusobacteria, and Spirochaetes, among which the most widespread genera include Streptococcus, Haemophilus, Neisseria, and Veillonella. $(9,10)$

$S$. salivarius is the most common species of streptococcus in the oral cavity of the newborns. Its abundance reaches its highest level at the age of three months, which is 10\%-15\% of the total number of streptococcal species. (11)

Research suggests that soft oral tissues can serve as a reservoir for pathogenic microorganisms, such as S. mutans. This finding is important for the prevention and oral hygiene before the eruption of the first teeth. $(9,10)$

Oral microbiome seems to be complete at the age of three but its maturation continues until adulthood. The bacterial flora of the oral cavity in the child varies during the development of teeth: deciduous, mixed, or permanent. The oral cavity microflora in children with deciduous teeth in relation to the other groups demonstrates a higher incidence of bacteria belonging to the following families: Pseudomonadaceae (Pseudomonas genus) Moraxellaceae (genera Acinetobacter Moraxella and Enhydrobacter), Enterobacteriaceae, and Pasteurellaceae (Aggregatibacter genus). During the replacement of deciduous teeth on the permanent ones the population of bacteria belonging to the family Veillonellaceae (genera Veillonella and Selenomonas) and Prevotella genus is subject to an increase, whereas bacteria of the family Carnobacteriaceae (Granulicatella genus) decrease. (12)

In addition to $S$. mutans other bacterial species are involved in the development and progression of dental caries, such as Lactobacillus, Veillonella, Bifidobacterium, Propionibacterium, acidic species independent of S. mutans (S. gordonii, S. oralis, S. mitis, and S. anginosus, Actinomyces, and Atopobium). $(11,12)$

Adolescence is a period of major hormonal changes, which also leads to a change in the composition of the oral microbiome. It is a multiplication of some groups of microorganisms, including gram-negative anaerobic bacteria and spirochetes. This change may be associated with an increased incidence of gingivitis.

The formation of a complete oral microbiome takes several years. In the case of the microbiome of the oral mucosa, this process can be considered to be completed after the eruption of the permanent teeth. (12) 


\section{Oral microbiome development in early childhood and influencing factors}

Predominate in ammonic fluid

- Streptococcus

- Fusobacterium

- Neisseria

- Prevotella

- Porphyromonas

The placental microbiome resembles closer to the pregnant mother's oral microbiome instead of the gut micrabiome.
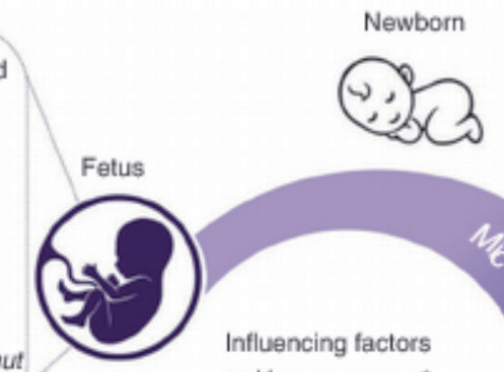

Influencing factors

- Human gemes*

- Pregnancy term

- Delivery method*

- Feeding method*

- Maternal

Primary dentition

Predominate

- Streptococcus mutans

- Fusobacteria

- Tenericutes

- synergistetes

- TM7

- SRT

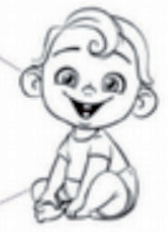

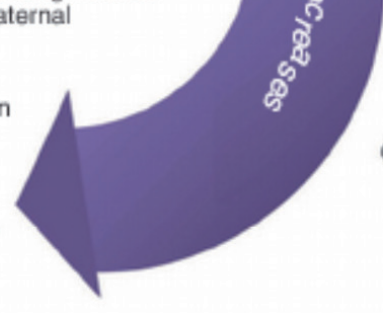

Early colonizer ( $0-3$ months)

- Streptococcus

- Streptococcus epidermidis

- Streptococcus salivarius

- Staphylococcus

- Fusobacterium

- Veillonella

- Lactobacillus

- Candida

Second colonizer (3-6 months)

- Gemella

- Granulicatella

- Haemophilus

- Rothia

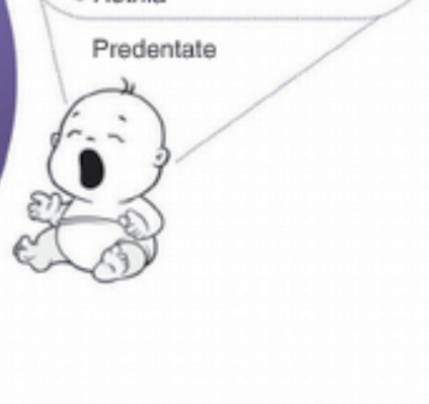

Human genes (influencing factor)

- One locus on chromosome 7 near gene IMMPL2

- One locus on chromosome 12 near gene INHBA-AS1

- Gene STAT3

Fig. 1 Oral microbiome development in early childhood and influencing factors (11)

\section{ROLE OF ORAL MICROBIOME}

Tooth decay, periodontal disease, and oral candidiasis are diseases that are caused by a dysbiosis of the oral microbiome. Two parameters are of a great importance for the health of the oral cavity: the $\mathrm{pH}$ of the environment of biofilms on the tooth surface and the degree of inflammatory response of the tissues that are in contact with these biofilms. (5)

Commensal microorganisms in the oral cavity play an important role in maintaining oral and systemic health. The presence of these microbes in the mouth suppresses colonization by pathogens. Since all oral surfaces are populated with them, few binding sites for pathogens remain. However, if this commensal flora is disturbed by antimicrobials, an overgrowth occurs (e.g. Candida).

Some bacteria are also antagonists of the oral pathogen. S. salivarius strain K12 produces bacteriocin that inhibits the growth of gram-negative bacteria that cause periodontitis and halitosis.

Gram-negative anaerobic Veillonella utilize the acids produced by streptococci, thereby inhibiting their demineralizing potential. In this way, acetate is formed, which is a substrate for the growth of Eubacterium alactolyticum. Of the gram-negative cocci, neisseria is interes- 
ting from the point of view of plaque formation, which, thanks to the formation of extracellular polysaccharides, contributes to the colonization of the tooth surface and also enables the attachment of microbes without adhesive capabilities.

However, S. sanguinis has a positive effect on the ecology of the oral cavity in terms of a possible damage to oral tissues as it competitively limits the colonization of $S$. mutans. In addition, S. sanguinis produces hydrogen peroxide which is lethal to Aggregatibacter (A.) actinomycetemcomitans. $(4,13,14)$

Candidiasis is a disease caused by an overgrowth of Candida (C.) albicans. These are usually superficial diseases. Candida is transmitted without symptoms to about half of the population and can also cause a number of acute and chronic infections in people who have reduced immunity, who are on immunosuppressive therapy after transplantation, HIV positive patients, as well as people with Down syndrome. $(3,4,5)$

In infant oral cavity the abundant and prevalent taxa were C. parapsilosis, C. tropicalis, S. cerevisiae, C. orthopsilosis, C. albicans, and Cladosporium velox. (11)

Table 1 Overview of oral bacteria (3)

\begin{tabular}{|l|l|}
\hline G+ bacteria & G- bacteria \\
\hline - Streptococcus & - Veilonella \\
- Mikrococcus & - Aggregatibacter \\
- Peptostreptococcus & - Neisseria \\
- Actinomyces & - Capnocytophaga \\
- Lactobacillus & - Centipeda \\
- Arachnia & - Moraxella \\
- Propionibacterium & - Haemophilus \\
- Eubacterium & - Bacteroides \\
- Rothia & - Eikenella \\
- Bifidobacterium & - Fusobacterium \\
- Bacterionema & - Leptotrichia \\
& - Treponema \\
& - Selenomonas \\
& - Wolinella \\
\hline
\end{tabular}

\section{DENTAL PLAQUE}

Dental plaque is a soft deposit that is formed on the surface of the teeth within 12-24 hours when the teeth are not cleaned. It is also called dental microbial biofilm - a highly organized community of various bacteria that adhere to a certain surface. It contains the bodies of microorganisms, an intermicrobial substance, and a fluid. The intermicrobial substance consists of glycoproteins from saliva and gingival exudate, extracellular polysaccharides, peeled epithelia, food scraps, and dead cells.

The development of the plaque takes 10-14 days in terms of quality and 3-4 days in terms of quantity. Within a period of 14 days without oral hygiene the development of microbial flora is completed.

Bacteria such as S. mutans, S. mitis, and S. salivarius are also called cariogenic and their amount in the oral cavity affects the number of carious lesions. The amount of Lactobacilli determines the rate of caries formation. The pathogenicity of dental plaque depends on several factors: adherence and virulence of bacteria, plaque retention in places that are inaccessible to purification, defense factors, the ability of bacteria to exchange genetic information, mineralization, and time factors. $(15,16,17)$ 
Studies performed by molecular biology methods show five dominant phyla of bacteria in the dental plaque: Firmicutes, Actinobacteria, Bacteroidetes, Fusobacteria, and Proteobacteria. These bacteria make up approximately $80 \%-95 \%$ of the oral microbiome. (12)

Research shows that the following four phyla of bacteria: Firmicutes, Bacteriodetes, Proteobacteria, and Actinobacteria, were present in deciduous, mixed, and permanent dentition. In deciduous dentition the proportion of Proteobacteria (Gammaproteobacteria, Moraxellaceae) was higher than Bacteroidetes, while in mixed and permanent dentitions their proportions were almost the same. Bacteroidetes (mainly genus Prevotella), Veillonellaceae family, Spirochaetes, and candidate division TM7 increased with the age of children, indicating the maturation of the oral microbiome caused by biological changes during adolescence. (18)

\section{PSYCHICAL DISEASES AND THEIR MANIFESTATIONS IN THE OROFACIAL AREA}

Disabled people are mainly people with congenital permanent disabilities (e.g. cerebral palsy, autism, Down syndrome), people with mental retardation, but also people with a combination of mental and physical disabilities. In addition to mental disabilities such people also have other associated illnesses that complicate their health, such as congenital heart disorders, epilepsy, allergies, and others.

\section{SUSCEPTIBILITY TO TOOTH DECAY}

In somatically and mentally handicapped individuals caries is affected by the same factors as in healthy people but there are also specific factors. Interesting are the findings of intact teeth, respectively low caries in some syndromes unless modified by dietary habits. These include various forms of microdontics with gaps where self-purification is possible (e.g. Down's syndrome).

On the contrary, there is increased caries in disorders of mineral metabolism (especially calcium). (19)

\section{PERIODONTAL DISEASES}

An increased incidence has been reported, especially in mentally retarded children, as early diagnosis is not possible due to difficult communication. Subsequently, the occurrence of these diseases was also found in adults.

Table 2 The most common causes of periodontal diseases in mentally retarded children (19)

Insufficient oral hygiene

Somatic diseases (diabetes mellitus)

Part of the syndrome (Down syndrome)

Orofacial involvement: oral closure mouth breathing untreated orthodontic defects

Insufficient ability of an individual to take care of his/her teeth

Lack of interest and insufficient motivation of the patient and parents

Early therapy of these diseases is important, which allows the preservation of functional teeth for as long as possible. In patients with a mental retardation this is complicated due to their inability to cooperate, so it is necessary to modify some therapeutic procedures. $(3,19)$ 


\section{ORAL MICROBIOME OF PERMANENTLY MENTALLY DISABLED CHILDREN}

Autism spectrum disorder (ASD) is associated with abnormalities such as buccal sensory sensitivity, taste and texture aversions, speech apraxia, and changes in salivary ribonucleic acid expression. Children with autism can have problems with oral hygiene and dental treatment is difficult to perform. In a study of children with ASD a higher caries prevalence, poor oral hygiene, and extensive unmet needs for dental treatment compared to the controls without autism were reported. $(20,21)$

The transcriptional activity of the salivary and dental microbiota in ASD patients differed markedly from that of healthy children. In children with ASD a lower bacterial diversity was demonstrated than in healthy chidren, consistent with findings from the gut. This finding was particularly pronounced in dental plaque samples. The genera Haemophilus in saliva and Streptococcus in dental plaque were in much greater numbers in ASD, while the incidence was reduced in Prevotella, Selenomonas, Actinomyces, Porphyromonas, and Fusobacterium.

Porphyromonas (P.) gingivalis causes dysbiosis of the periodontal microflora. In patients with a localized chronic periodontitis the transmission of oral bacteria to the gut may occur, resulting in microbial dysregulation in the gut. Changes in the gut microbiota composition could induce the permeability of the gut barrier and an immune activation leading to a systemic inflammation.

Microbial dysbiosis may be influenced by insuficient oral hygiene, lack of a varied diet, and placing objects into the mouth. The importance of regular oral hygiene is necessary for the maintenance of oral homeostasis. Furthermore, dental caries can be caused by ecological imbalance of commensal microbiota (mainly due to lack of a varied diet, such as frequent carbohydrate consumption). Placing foreign objects in the mouth is yet another source of dysbiosis because these objects can be contaminated with microorganisms from unwashed hands in contact with other human body fluids. $(22,23)$

In a study on adolescents with Down syndrome (DS), an changed microbial composition of subgingival plaque was observed, with a higher frequency of A. actinomycetemcomitans, Capnocytophaga and $P$. gingivalis detected. Other studies point out that children with DS experience a very early colonization of various periodontopathic bacteria, with a higher prevalence of species such as $P$. gingivalis, Tannerella (T.) forsythia and Treponema denticola. In the subgingival microbiota of children with DS the presence of $T$. forsythia and Actinomyces naeslundii was observed. At older ages the patients presented with P. gingivalis, A. actinomycetemcomitans, Campylobacter rectus, Eikenella corrodens, Prevotella (P.) intermedia, P. nigrescens, Capnocytophaga sputigena, and Peptostreptococcus micros. The results indicate that children and adolescents with DS have a higher susceptibility to periodontal changes and a higher prevalence and density of some periodontal pathogens. $(24,25)$

The research shows that oral and gingival bacterial flora of the studied mentally retarded people was significantly different from oral bacterial flora in the mentally normal people, which is justified considering their clinical differences and their insuficient oral hygiene. The higher occurrence of Pneumococcus in these people than that in mentally healthy people increases the risk of bacterial respiratory infections. In this study the prevalence of bacterial oral microbiome has a significant relationship with oral hygiene of these people. With an improvement in oral health care we can decrease these unwanted changes. (26)

\section{CONCLUSION}

Several studies show that the oral microbiomes of healthy and permanently mentally disabled children differ. The disabled children have a higher susceptibility to periodontal disease and a number of periodontopathic bacteria. A higher risk of dental caries is described, which could be attributed to difficulties in performing adequate oral hygiene. For this 
group of children we recommend daily plaque removal, a reduction in the amount and frequency of sugar intake, use of fluoride varnishes, and stimulation of saliva.

The treatment of pediatric patients with permanent mental disabilities is often radical. Unwanted extractions of teeth often occur due to an extensive tooth destruction and odontogenic inflammatory processes. Therefore, it is necessary to implement the best possible preventive measures to prevent premature tooth loss in children. Due to their general disease, prosthetic tooth replacements often cannot be made for them and, thus, the processing of food in the oral cavity is impaired. This, of course, also affects the entire digestive process. The aim is to improve the quality of life of these pediatric patients by optimizing preventive-therapeutic procedures.

In addition to all this it is very important to appeal to parents not to neglect the care of their children's oral cavity. Parents are responsible for the health of the children's oral cavity. Children are not capable enough to remove dental plaque from tooth surfaces and the interdental spaces. The quality of oral hygiene performed by children is not sufficient and tooth decay and periodontal diseases occur. Therefore, it is necessary for parents to thoroughly clean their children's teeth and motivate children to take care of their oral cavity.

\section{REFERENCES}

1. Lederberg J, Mccray AT. 'Ome Sweet 'Omics-- A Genealogical Treasury of Words. The Scientist. 2001; 15 (7): 8. Available from: https://lhncbc.nlm.nih.gov/system/files/pub2001047.pdf

2. Kilian J. Prevence ve stomatologii. Praha: Galen; 1999.

3. Pramod JR. Textbook of oral medicine. New Delhi: Jaypee Brothers Medical Publishers; 2014.

4. Wade WG. The oral microbiome in health and disease. Pharmacol Res. 2013; 69 (1): 137-143.

5. Kilian M. The oral microbiome - friend or foe?. Eur J Oral Sci. 2018; 126 (Suppl. 1): 5-12. Available from: https://onlinelibrary.wiley.com/doi/full/10.1111/eos.12527

6. Gomez A, Nelson KE. The Oral Microbiome of Children: Development, Disease and Implications Beyond Oral Health. Microb Ecol. 2017; 73 (2): 492-503. Available from: https://www.ncbi.nlm.nih.gov/pmc/articles/PMC5274568/

7. Dominguez-Bello MG, Costello EK, Contreras M, Magris M, Hidalgo G, Fierer N, Knight R. Delivery mode shapes the acquisition and structure of the initial microbiota across multiple body habitats in newborns. Proc Natl Acad Sci U S A. 2010; 107 (26): 11971-11975. Available from: https:// www.ncbi.nlm.nih.gov/pmc/articles/PMC2900693/

8. Srivastava M, Masih U, Kour G, Saini M, Rathore K. Microbiota of new born infant of caesarean \& vaginaldeliveries: a comparative microbiological study. Int J Adv Res. 2017; 5 (4): 2017-2022. Available from: file:///d:/Downloads/ORAL_MICROBIOTA_OF_NEW_BORN_INFANT_OF_CAESAREAN_VA.pdf

9. Cephas KD, Kim J, Mathai RA, Barry KA, Dowd SE, Meline BS, Swanson KS. Comparative Analysis of Salivary Bacterial Microbiome Diversity in Edentulous Infants and Their Mothers or Primary Care Givers Using Pyrosequencing. PLos One. 2011; 6 (8): e23503. Available from: https://www. ncbi.nlm.nih.gov/pmc/articles/PMC3154475/

10. Sampaio-Maia B, Monteiro-Silva F. Acquisition and maturation of oral microbiome throughout childhood: An update. Dent Res J. 2014; 11 (3): 291-301. Available from: https://www.ncbi.nlm.nih.gov/pmc/articles/PMC4119360/

11. Xiao J, Fiscella KA, Gill SR. Oral microbiome: possible harbinger for children's health. Int J Oral Sci. 2020; 12 (1): 12. Available from: https://www.researchgate.net/publication/341049093_Oral_microbiome_possible_harbinger_for_children\%27s_health

12. Krzyściak W, Jurczak A, Piątkowski J. The Role of Human Oral Microbiome in Dental Biofilm Formation. In: Dhanasekaran D, Thajuddin N. Microbial Biofilms: Importance and Applications. Rijeka: InTech; 2016. p. 329-381. Available from: https://www.intechopen.com/books/microbial-biofilmsimportance-and-applications/the-role-of-human-oral-microbiome-in-dental-biofilm-formation 
13. Lindhe J, Thorkild K, Lang N. Clinical periodontology and implant dentistry. Copenhagen: Boisen print; 2000.

14. Kreth J, Merritt J, Shi W. Competition and Coexistence between Streptococcus mutans and Streptococcus sanguinis in the dental biofilm. J Bacteriol. 2005; 187 (21): 7193-7203. Available from: https://www.ncbi.nlm.nih.gov/pmc/articles/PMC1272965/

15. Minčík J, Šatánková M, Alexejenko M. Kariológia. Košice: JES; 2014.

16. Weber T. Memorix zubniho lekarstvi. Praha: Grada; 2012.

17. Lamont RJ, Hajishengallis G, Jenkinson HF. Oral microbiology and immunology. Washington, DC: ASM Press; 2014.

18. Crielaard W, Zaura E, Schuller AA, Huse SM, Montijn RC, Keijser BJF. Exploring the oral microbiota of children at various developmental stages of their dentition in the relation to their oral health. BMC Med Genomics. 2011; 4: 22. Available from: https://www.ncbi.nlm.nih.gov/pmc/ articles/PMC3058002/

19. Nováková K. Stomatologicka pece o hendikepovane pacienty. Olomouc: Univerzita Palackeho v Olomouci; 2008.

20. Olsen I, Hicks SD. Oral microbiota and autism spectrum disorder (ASD). J Oral Microbiol. 2020; 12 (1): 1702806. Available from: https://www.ncbi.nlm.nih.gov/pmc/articles/PMC6913665/

21. Jaber MA. Dental caries experience, oral health status and treatment needs of dental patients with autism. J Appl Oral Sci. 2011; 19 (3): 212-217. Available from: https://www.ncbi.nlm.nih. gov/pmc/articles/PMC4234331/

22. Hicks SD, Uhlig R, Afshari P, Williams J, Chroneos M, Tierney-Aves C, Wagner K, Middleton FA. Oral Microbiome Activity in Children With Autism Spectrum Disorder. Autism Res. 2018; 11 (9): 1286-1299. Available from: https://beta.quadrantbiosciences.com/wp-content/uploads/2019/ 10/Oral-microbiome-activity-in-children-with-autism-spectrum-disorder.pdf

23. Qiao Y, Wu M, Feng Y, Zhou Z, Chen L, Chen F. Alterations of oral microbiota distinguish children with autism spectrum disorders from healthy controls. Scientific Reports. 2018; 8: 1597. Available from: https://www.nature.com/articles/s41598-018-19982-y

24. Areias C, Sampaio-Maia B, Macho V, Norton A, Macedo P, Casimiro de Andrade D. Oral Health in Down Syndrome. In: Dey S. Health Problems in Down Syndrome. Rijeka: InTech; 2015. p. 45-68. Available from: https://www.intechopen.com/books/health-problems-in-down-syndrome/oralhealth-in-down-syndrome

25. Carrada CF, Scalioni FAR, Cesar DE, Devito KL, Ribeiro LC, Ribeiro RA. Salivary Periodontopathic Bacteria in Children and Adolescents with Down Syndrome. PLoS One. 2016; 11 (10): e0162988. Available from: https://www.ncbi.nlm.nih.gov/pmc/articles/PMC5058504/

26. Ahanjan M, Akhavan A, Abedian F, Mirabi AM. Study of oral and gingival microbial flora in institutionalized mentally retarded patients of sari- 2011. Afr J Cln Exper Microbiol. 2013; 14 (2): 56-61.

Received: November, 11, 2020

Accepted: November, 16, 2020 